'Grupo IRyS. Escuela de Educación Física. Pontificia Universidad Católica de Valparaíso, Chile.

2Departamento de Ciencias de la Actividad Física, Universidad de Los Lagos, Osorno, Chile.

${ }^{3}$ Centro de Salud Familiar Tomás Rojas, Los Lagos. Chile.

${ }^{4}$ Facultad de Ciencias de la Actividad Física, Universidad San Sebastián, Concepción, Chile.

Instituto de Farmacia, Facultad de Ciencias, Universidad Austral de Chile, Valdivia, Chile.

${ }^{6}$ Grupo de Investigación Calidad de Vida Universidad del Biobío, Chillán, Chile. ${ }^{7}$ Instituto de Anatomía, Histología y Patología, Facultad de Medicina, Universidad Austral de Chile, Valdivia, Chile.

${ }^{8}$ Departamento de Educación Física. Facultad de Educación. Universidad de Concepción. Concepción, Chile.

'Unidad de Ergonomía, Facultad de

Ciencias Biológicas, Universidad de Concepción, Concepción, Chile.

${ }^{10}$ Departamento de Trabajo Social, INACAP, Valdivia, Chile.

"Departamento de Nutrición, Facultad de Farmacia, Universidad de Concepción, Concepción, Chile.

${ }^{2}$ Departamento de Educación Física Deportes y Recreación, Universidad de la Frontera, Temuco, Chile.

${ }^{13}$ BHF Glasgow Cardiovascular Research Centre, Institute of Cardiovascular and Medical Science, University of Glasgow, Glasgow, United Kingdoms. aMagíster. Educación Física.

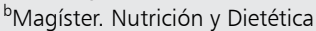
'Magíster. Educación. dMagíster. Neurociencias y Salud Mental. eTrabajadora Social. fMagíster. Nutrición. ${ }_{9}^{9}$ Magíster. Fisiología Clínica del Ejercicio. hPhD

Recibido el 15 de marzo de 2016 aceptado el 6 de julio de 2016 .

Correspondencia a:

Dr. Carlos Celis-Morales

BHF Glasgow Cardiovascular Research Centre.

University of Glasgow. Glasgow, United Kingdom. Tel: 44(0)1413304201 carlos.celis@glasgow.ac.uk

\section{Fitness cardiorrespiratorio se asocia a una mejora en marcadores metabólicos en adultos chilenos}

\author{
CARLOS CRISTI-MONTERO ${ }^{1, \mathrm{~h}}$, RODRIGO RAMÍREZ-CAMPILLO ${ }^{2, \mathrm{~h}}$, \\ CRISTIAN ALVAREZ ${ }^{3, a}$, ALEX GARRIDO MÉNDEZ ${ }^{4, \mathrm{~h}}$, \\ MARÍA ADELA MARTÍNEZ ${ }^{5, b}, X_{\text {IMENA DÍAZ MARTÍNEZ }}^{6, c}$, \\ ANA MARÍA LEIVA ${ }^{7, d}$, CARLOS SALAS ${ }^{8, a}$, \\ MANUEL GUTIÉRREZ ${ }^{9, h}$, RUTH SANZANA-INZUNZA $^{10, \mathrm{e}}$, \\ ELIANA DURÁN ${ }^{11, \mathrm{f}}$, ANA MARÍA LABRAÑA ${ }^{11, \mathrm{f}}$, \\ NICOLÁS AGUILAR-FARÍAS ${ }^{12, \mathrm{~g}, \mathrm{~h}}$, CARLOS CELIS-MORALES ${ }^{13, \mathrm{~h}}$
}

\section{Inverse association of cardiorespiratory fitness with cardiovascular risk factors in Chilean adults}

Background: A high level of cardiorespiratory fitness (CRF) is an important protector against cardiovascular and metabolic diseases. Aim: To explore the association of CRF with several metabolic markers and estimate the variation of these parameters by an increase of 1 MET change in CRF. Material and Methods: Cross-sectional study of 447 adults (56\% women) without medical history of cardiometabolic diseases. Physical activity evaluated using accelerometry, body mass index (BMI), blood pressure, blood glucose and insulin and lipid profile were measured. HOMA-IR was calculated with fasting glucose and insulin levels. The submaximal Chester Step Test was used to measure CRF. Results: CRF was significantly associated with the level and intensity of physical activity, and all metabolic markers ( $p$-trend $<0.05)$, except with diastolic blood pressure. It was estimated that 1-MET increase in CRF is associated with waist circumference $(\beta-0.32 \mathrm{~cm})$, fat mass $(\beta-0.22 \%)$, insulin $(\beta-0.67$ pmol. $\left.\mathrm{l}^{-1}\right)$, HOMA-IR $(\beta-0.17)$ and HDL cholesterol $\left(\beta+1.46 \mathrm{mg} . \mathrm{dl}^{-1}\right)$. All these associations were independent of main covariates such as age, sex, education, smoking and body mass index. Conclusions: A higher level of CRF is inversely associated with several metabolic markers that are risk factors for cardiovascular disease.

(Rev Med Chile 2016; 144: 980-989)

Key words: Body Composition; Diabetes Mellitus; Exercise; Fitness; Obesity; Physical.
1 1 fitness cardiorrespiratorio (FitCR) es un importante indicador fisiológico relacionado con la capacidad máxima de una persona para consumir oxígeno $\left(\mathrm{VO}_{2} \max \right)$ como respuesta a las demandas energéticas del organismo ${ }^{1}$. Actual- mente, recomendaciones internacionales sugieren realizar al menos $150 \mathrm{~min}$ a la semana de actividad física de intensidad moderada a vigorosa, o 75 min a la semana de actividad física de intensidad vigorosa, o bien, la combinación de ambas con 
el fin de mantener o incrementar el FitCR ${ }^{1}$. Sin embargo, gran parte de la población chilena y del mundo no cumple estas recomendaciones ${ }^{2,3}$.

Cabe señalar que Chile, al igual que el resto de los países latinoamericanos, presenta altos niveles de inactividad física y sedentarismo $(18,9 \%$ y $35,9 \%$, respectivamente $)^{3,4} \mathrm{y}$ ambos componentes, han demostrado actuar negativamente y de forma independiente el uno del otro sobre el FitCR ${ }^{5}$.

Existe suficiente evidencia científica que demuestra la fuerte asociación entre el FitCR con la prevención y el tratamiento de diversas enfermedades crónicas no transmisibles $(\mathrm{ECNT})^{6,7}$. El fundamento subyacente respecto a los beneficios en la salud al aumentar el FitCR se centrarían principalmente a una mayor eficiencia metabólica a consecuencia de un aumento del número y volumen mitocondrial ${ }^{8}$, a un aumento del gasto cardiaco ${ }^{9}$, entre otros ${ }^{7}$.

Se ha estimado que un incremento de sólo 1-MET (equivalente metabólico $=\sim 3,5 \mathrm{mlO} \cdot \mathrm{kg}^{-1}$. $\mathrm{min}^{-1}$ ) es capaz de reducir en un $15 \%$ el riesgo de desarrollar enfermedades cardiovasculares (ECVs) $\mathrm{y}$ en un $13 \%$ el riesgo de mortalidad global ${ }^{10}$. Adicionalmente, el FitCR reduciría la comorbilidad y mortalidad de forma independiente a otros marcadores clásicos de salud tales como el índice de masa corporal (IMC), porcentaje de grasa y perímetro de cintura ${ }^{11-13}$. Por tanto, el primer objetivo del presente estudio fue establecer la asociación entre el nivel de FitCR y diversos marcadores metabólicos, y el segundo, estimar la variación de estos parámetros por un aumento de un equivalente metabólico (1-MET), como una posible estrategia de salud.

\section{Material y Método}

\section{Diseño del estudio}

Este es un estudio observacional analítico de corte transversal que utiliza información del proyecto GENADIO (Genes, Ambiente, Diabetes y Obesidad) realizado en Chile entre los años 2009-2011. El estudio GENADIO tiene como objetivo evaluar la prevalencia de factores de riesgo de ECVs en Chile, y cuenta con la aprobación de los comités de ética de la Universidad de Chile, Universidad de Concepción y Universidad de Glasgow (Reino Unido). Todos los participantes firmaron su consentimiento informado previo a la recolección de datos.
La población estudiada fue compuesta por residentes de las regiones del Biobío y Los Ríos, sin historial médico de enfermedad metabólica o cardiovascular, y que al momento de la evaluación no estuvieran bajo ninguna prescripción de medicamentos, estos criterios han sido mencionado anteriormente ${ }^{14}$. El cálculo del tamaño muestral y los criterios de inclusión han sido descritos en extenso en publicaciones previas ${ }^{14}$. Los criterios de exclusión fueron (a) residencia en zona rural o urbana por un período menor a 2 años $(n=13)$, (b) personas que presentaron problemas de salud mental y que no dieron el consentimiento informado $(n=2)$, (c) personas en etapa de embarazo $(\mathrm{n}=4)$ y $(\mathrm{d})$ personas que no quisieron participar del estudio $(n=187)$. Fueron incluidas finalmente 447 personas. Los datos sociodemográficos fueron recolectados mediante encuestas validadas ${ }^{14}$.

\section{Medición del fitness cardiorrespiratorio (FitCR) y actividad física}

El Chester Step Test fue utilizado para la medición de FitCR expresada en METs, el cual es un test progresivo submaximal de tres etapas, que utilizó la frecuencia cardiaca medida con monitor Polar $\left(\mathrm{S} 810^{\mathrm{TM}}, \mathrm{US}\right)$, altura del escalón, sexo y edad para estimar el $\mathrm{VO}_{2}$ max, $y$ ha sido previamente validado ${ }^{15}$. La actividad física y tiempo sedentario fueron medidos con acelerómetros de movimiento (Actigraph GTM1, US). Los participantes fueron instruidos para utilizar el acelerómetro en su cadera izquierda, para lo cual se les entregó una banda elástica que les permitiría fijar el monitor en esa zona. El acelerómetro se utilizó durante 7 días consecutivos, y sólo se removió durante las horas destinadas a dormir, ducharse o realización de actividades acuáticas. Para que un día fuera valido el acelerómetro debía registrar al menos $10 \mathrm{~h}$ de datos válidos, y para que la persona fuera incluida en el estudio debía presentar al menos 3 días válidos de la semana. Tanto la intensidad de la actividad física como el gasto energético (expresado en MET. $\mathrm{h}^{-1}$.semana ${ }^{-1}$ ) fueron determinados mediante el algoritmo de Freedson ${ }^{16}$.

\section{Mediciones antropométricas y metabólicas}

La evaluación antropométrica se realizó por personal capacitado utilizando protocolos estandarizados ${ }^{17}$. El peso y la talla fueron determinados con una balanza electrónica (TANITA TBF 300A, USA) y tallímetro (SECA A800, USA) con una 
precisión de 100 g y $1 \mathrm{~mm}$, respectivamente. El perímetro de cintura fue medido con una cinta métrica no distendible (SECA Modelo 201, US). El IMC fue calculado usando la ecuación de [peso/ talla ${ }^{2}$ y el estado nutricional se definió mediante los valores de corte de la Organización Mundial de la Salud. Los valores utilizados para definir obesidad central fueron los siguientes: perímetro de cintura $\geq 102 \mathrm{~cm} \mathrm{y} \geq 88 \mathrm{~cm}$ en hombres y mujeres, respectivamente. La composición corporal se determinó mediante la medición de cuatro pliegues cutáneos (bíceps, sub-escapular, supra-iliaco y tríceps) ${ }^{14}$ y se aplicó el algoritmo de Durnin y Womersley para estimar el porcentaje de masa grasa ${ }^{18}$.

Para la determinación de las variables metabólicas se obtuvieron muestras de sangre venosa tras un ayuno de 8 a $12 \mathrm{~h}$. Los valores de glicemia, colesterol total (CT), triglicéridos (TG) y colesterol HDL (c-HDL) fueron determinados mediante métodos enzimáticos colorimétricos ${ }^{14}$. El colesterol LDL (c-LDL) fue determinado con la ecuación de Friedewald ${ }^{19}$. La insulina fue determinada mediante la técnica de radio-inmunoensayo, mientras que la resistencia a la insulina fue determinada mediante la ecuación de HOMA-IR ${ }^{20}$. Todas las mediciones fueron realizadas en laboratorios certificados y mediantes técnicas estandarizadas de la Universidad de Chile y la Universidad de Glasgow, $\mathrm{y}$ han sido descritos previamente $\mathrm{e}^{14,21}$.

Finalmente, la presión arterial se determinó con el promedio de dos mediciones realizadas después de estar 10 min en reposo, utilizando un monitor validado (OMRON HEM705, UK) ${ }^{14}$.

\section{Análisis de datos}

Se utilizó estadística descriptiva para caracterizar variables de interés según tertiles de FitCR. La distribución normal de las variables fue analizada mediante el test de Anderson Darling y Kruskal-Wallis. Las diferencias entre grupos para variables cuantitativas fue estimada mediante General Linear Model (GLM) y las diferencias de prevalencias entre grupos de FitCR fue estimada mediante $\chi^{2}$. La asociación entre FitCR y las variables de tipo metabólicas fue determinada mediante regresión múltiple, ajustando los modelos estadísticos por variables confundidoras (edad, sexo, nivel educacional, hábito de fumar). Las variables metabólicas fueron también ajustadas por IMC y transformadas a Z-score para el análisis de comparación. Los valores de tendencia para FitCR en variables de interés fueron determinados mediante regresión lineal. La correlación entre FitCR e intensidades de actividad física fue determinada mediante el test de Pearson. Valor $\mathrm{p}$ aceptado para diferencias significativas fue $<0,05$. Todos los análisis fueron realizados con el software STATA SE 14.

\section{Resultados}

En la Tabla 1 se presentan las características sociodemográficas, antropométricas y de actividad física de los participantes según nivel de FitCR. Se destaca que los participantes con mayor nivel de FitCR son más jóvenes, tienen un menor porcentaje de masa grasa, son más activos y destinan menos tiempo a actividades de tipo sedentarias. No se observó una interacción significativa entre tertiles de FitCR y sexo $(p=0,438)$, por lo cual los resultados no se estratificaron.

El grado de asociación entre el FitCR, actividad física total, tiempo destinado a actividad física de intensidad ligera, moderada, vigorosa y sedentarismo son presentadas en la Figura 1. Se aprecia que sólo el tiempo sedentario muestra una correlación inversa con el FitCR ( $r=-0,413, p<0,05)$, mientras que todas las otras variables presentan asociaciones positivas y significativas.

En las Figuras 2 y 3 se representan gráficamente el grado de asociación entre los marcadores antropométricos y marcadores metabólicos, respectivamente, según tertiles de FitCR. En la Figura2 se observa que tanto el perímetro de cintura como el porcentaje de grasa muestran una relación negativa a medida el FitCR es mayor ( $\mathrm{p}$-trend $<0,05$ ). Mientras que todos los marcadores metabólicos (presión arterial sistólica, glicemia, insulina, HOMA-IR, TG, CT, c-HDL yc-LDL) a excepción de la presión arterial diastólica muestran la misma tendencia que los marcadores antropométricos (Figura 3).

La Figura 4 presenta la estimación en la variación de los marcadores antropométricos y metabólicos por cada 1-MET de incremento en el FitCR. Los resultados demuestran que todas las variables estudiadas (excepto IMC y presión arterial diastólica) presentan una asociación inversa tanto para sus valores de medida $\beta$ como para su Z-score (p-trend $<0,05)$. 
Tabla 1. Características socio-demográficas, antropométricas y de actividad física según tertiles de FitCR

\begin{tabular}{|c|c|c|c|c|}
\hline Variables & Bajo $(n=150)$ & $\begin{array}{l}\text { Tertiles de FitCR } \\
\text { Medio }(n=149)\end{array}$ & Alto $(n=148)$ & Valor $\mathbf{p}$ \\
\hline $\begin{array}{l}\text { Socio-demográficas } \\
\text { Edad (años) } \\
\text { Sexo (mujeres) (\%) }\end{array}$ & $\begin{array}{c}40,9 \pm 14,2 \\
56,0\end{array}$ & $\begin{array}{c}37,1 \pm 12,2 \\
56,3\end{array}$ & $\begin{array}{c}33,5 \pm 10,7 \\
56,0\end{array}$ & $\begin{array}{c}<0,0001 \\
0,998\end{array}$ \\
\hline $\begin{array}{l}\text { Nivel educacional (\%) } \\
\text { Básica } \\
\text { Media } \\
\text { Superior }\end{array}$ & $\begin{array}{l}18,49 \\
44,52 \\
36,99\end{array}$ & $\begin{array}{l}22,07 \\
45,52 \\
32,41\end{array}$ & $\begin{array}{l}50,68 \\
31,76 \\
17,57\end{array}$ & $<0,0001$ \\
\hline $\begin{array}{l}\text { Antropométricas } \\
\text { Peso }(\mathrm{kg}) \\
\text { Talla }(\mathrm{cm}) \\
\mathrm{IMC}\left(\mathrm{kg} / \mathrm{m}^{2}\right)\end{array}$ & $\begin{array}{c}73,9 \pm 11,6 \\
160,1 \pm 9,4 \\
28,8 \pm 3,9\end{array}$ & $\begin{array}{c}69,8 \pm 9,9 \\
158,9 \pm 8,0 \\
27,7 \pm 3,7\end{array}$ & $\begin{array}{c}70,5 \pm 8,9 \\
158,2 \pm 7,3 \\
28,2 \pm 3,5\end{array}$ & $\begin{array}{l}0,989 \\
0,054 \\
0,168\end{array}$ \\
\hline $\begin{array}{l}\text { Estado nutricional (\%) } \\
\text { Normal } \\
\text { Sobrepeso } \\
\text { Obeso }\end{array}$ & $\begin{array}{l}18,67 \\
36,00 \\
45,33\end{array}$ & $\begin{array}{l}23,49 \\
23,49 \\
53,02\end{array}$ & $\begin{array}{l}16,22 \\
29,73 \\
54,05\end{array}$ & 0,118 \\
\hline Masa grasa (\%) & $30,6 \pm 3,9$ & $29,2 \pm 5,1$ & $28,4 \pm 4,8$ & $<0,0001$ \\
\hline Perímetro de cintura (cm) & $98,9 \pm 11,1$ & $96,5 \pm 10,6$ & $97,9 \pm 10,9$ & 0,732 \\
\hline Obesidad central (\%) & 64,0 & 61,0 & 64,1 & 0,823 \\
\hline $\begin{array}{l}\text { FitCR y Actividad física } \\
\text { FitCR (METs) } \\
\text { Actividad física (MET. } \mathrm{min}^{-1} \mathrm{sem}^{-1} \text { ) } \\
\text { Físicamente inactivo }(\%) \\
\text { Sedentarismo (min.dia }{ }^{-1} \text { ) }\end{array}$ & $\begin{array}{c}9,9 \pm 1,4 \\
5643 \pm 1888 \\
43,52 \\
529 \pm 98,3\end{array}$ & $\begin{array}{c}12,8 \pm 1,5 \\
6267 \pm 1985 \\
32,65 \\
494 \pm 91,0\end{array}$ & $\begin{array}{c}16,9 \pm 2,3 \\
7087 \pm 1966 \\
15,89 \\
431 \pm 85,6\end{array}$ & $\begin{array}{l}<0,0001 \\
<0,0001 \\
<0,0001 \\
<0,0001\end{array}$ \\
\hline
\end{tabular}

Datos presentados como media y desviación estándar para variables continuas, y como porcentaje para variables categóricas IMC: índice de masa corporal. FitCR: Fitness cardiorrespiratorio; sem: semana; min: minuto.

\section{Discusión}

Los principales hallazgos de este estudio evidencian una asociación inversa y significativa entre FitCR y diversos parámetros de adiposidad y metabólicos en adultos chilenos. Estas asociaciones fueron independientes de la edad, sexo, IMC y hábito de fumar. Dado el fuerte vínculo entre FitCR y marcadores de salud cardiovascular y mortalidad ${ }^{6,10-13}$, los resultados de esta investigación podrían ser de utilidad, para establecer la medición del FitCR como una herramienta de screening a nivel de salud pública con la intención de poder identificar tempranamente a la población con baja capacidad cardiorrespiratoria y por ende con mayor riesgo de desarrollar ECV y mortalidad.

En población adulta chilena son escasas las publicaciones que entreguen evidencia científica respecto a la influencia del FitCR sobre diversos marcadores relacionados con ECVs y salud metabólica. A nuestro entender, sólo Krämer et al., en el 2009 han demostrado una relación inversa entre diversos marcadores de salud en casi 2.000 chilenos sin antecedentes de ECVs ${ }^{22}$. No obstante, nuestro estudio también añade un aspecto práctico, necesario en medicina preventiva, ayudando a reducir la brecha entre el campo clínico y la salud pública. Es por esto que hemos indagado sobre la variación de los parámetros de estudio (antropométricos y metabólicos) con un posible incremento de 1-MET en el FitCR como una posible estrategia de salud.

Los resultados de esta investigación concuerdan con importantes estudios epidemiológicos prospectivos internacionales que han demostrado 

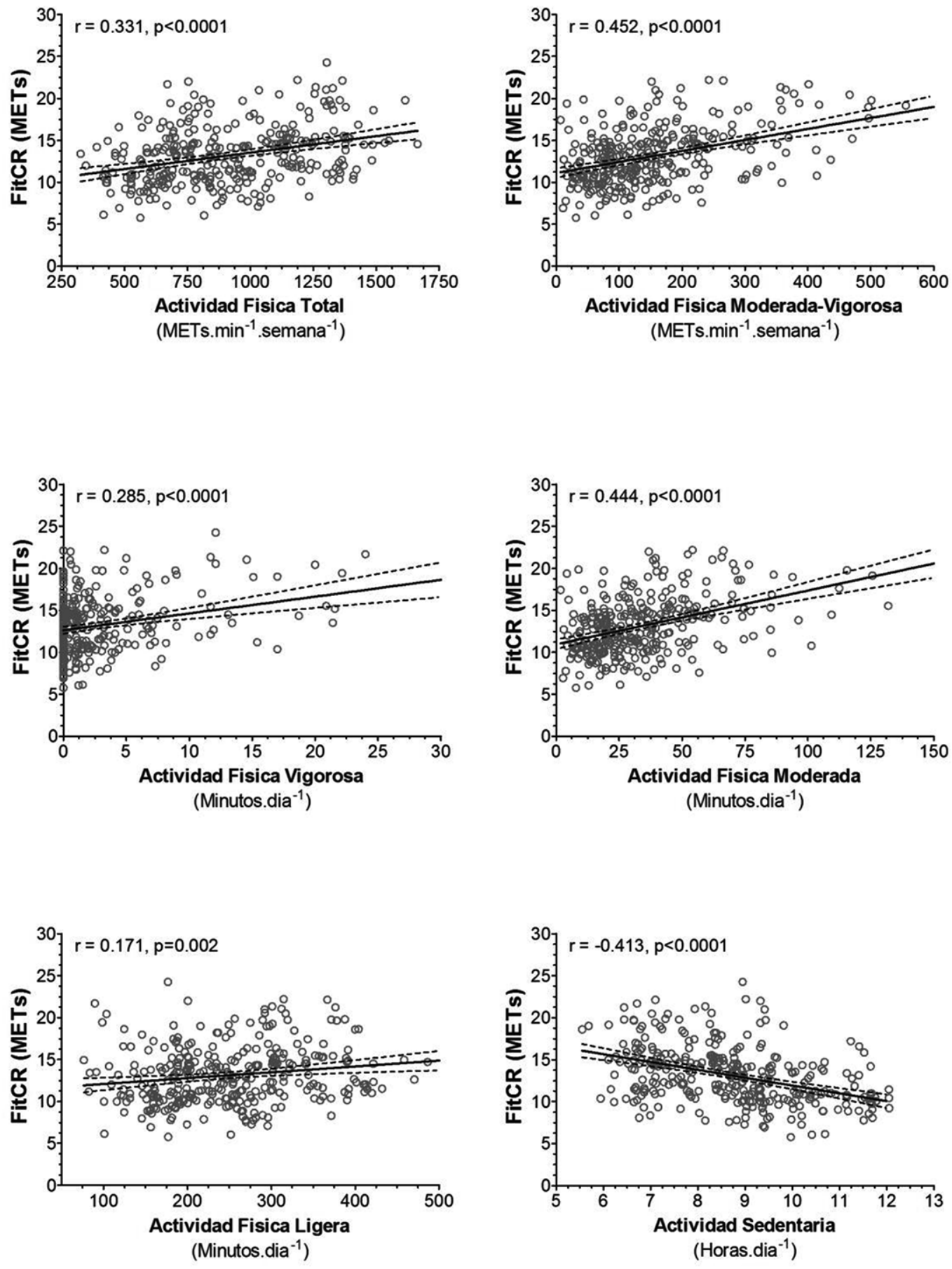

Figura 1. Correlación entre FitCR y diferentes intensidades de actividad física medidas objetivamente con acelerómetros de movimiento. La correlación entre variables fue estimada con el test de Pearson. 

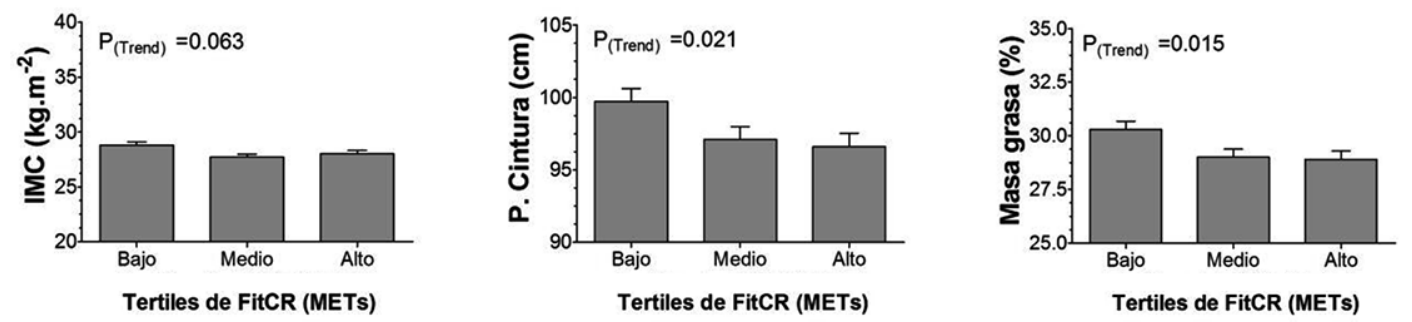

Figura 2. Asociación entre marcadores antropométricos según tertiles de FitCR. Datos son presentados como media y desviación estándar. Todos los análisis fueron ajustados por edad, nivel educacional, sexo y hábito de fumar.
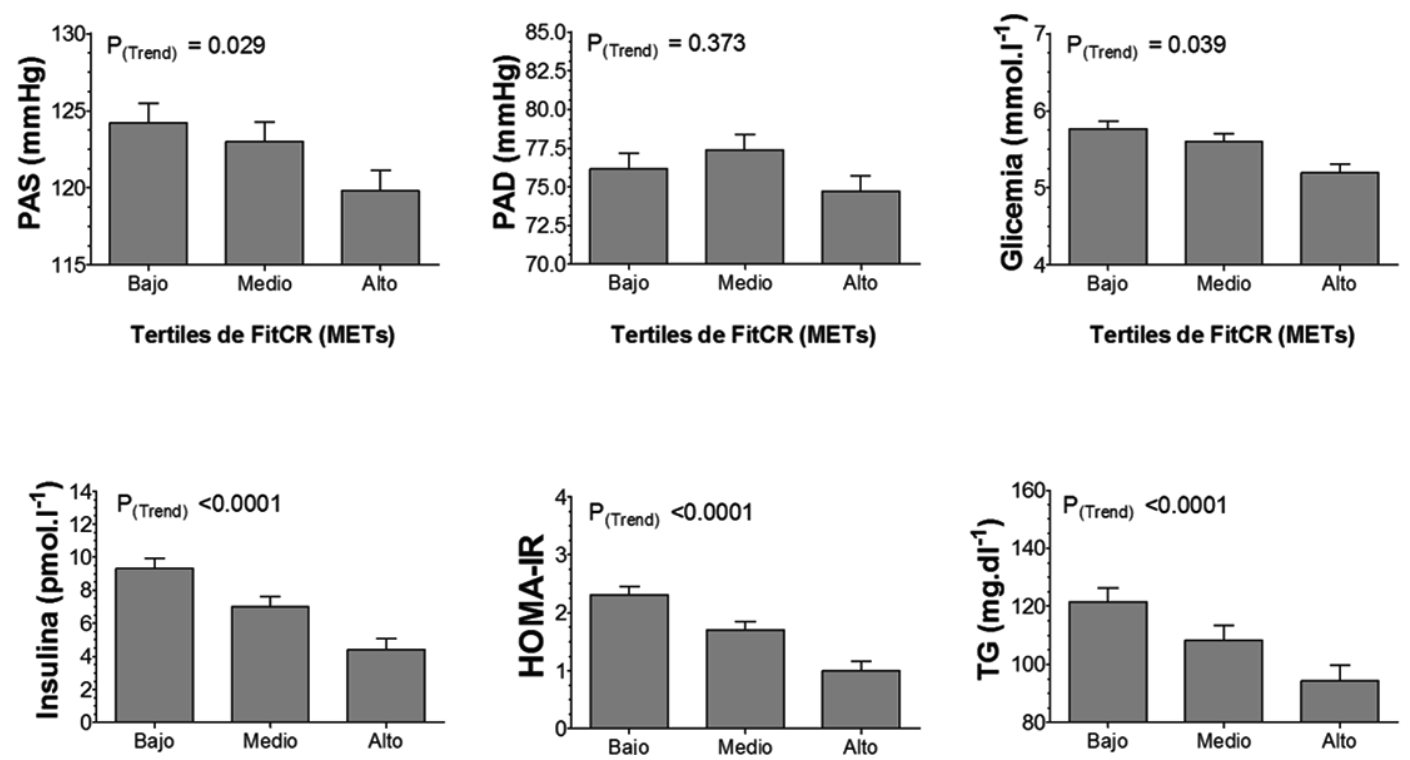

Tertiles de FitCR (METs)

Tertiles de FitCR (METs)

Tertiles de FitCR (METs)
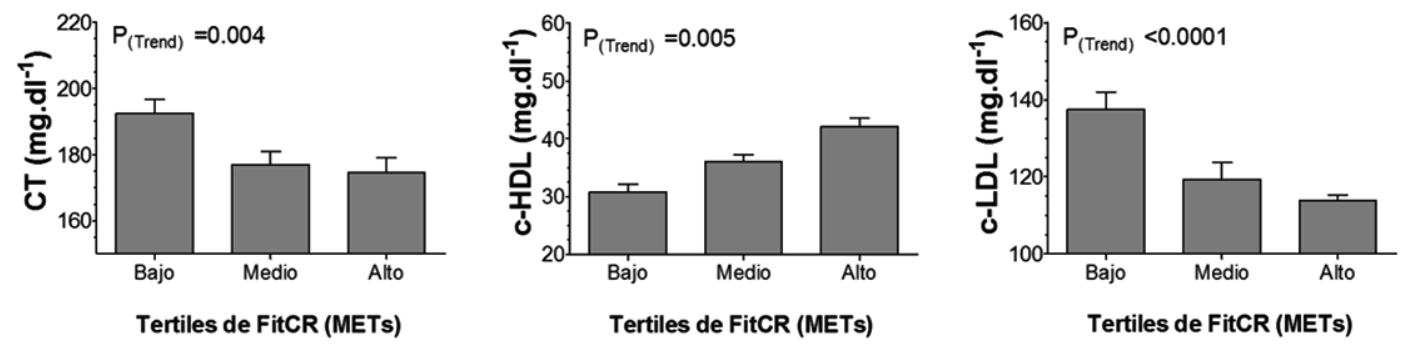

Figura 3. Asociación entre marcadores metabólicos según tertiles de FitCR. Los datos se presentan como media y desviación estándar. Todos los análisis fueron ajustados por edad, nivel educacional, sexo, hábito de fumar e IMC. CT: Colesterol Total. 


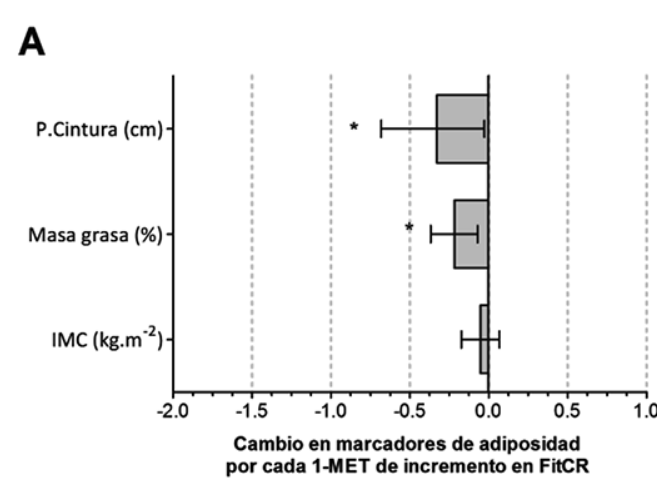

B

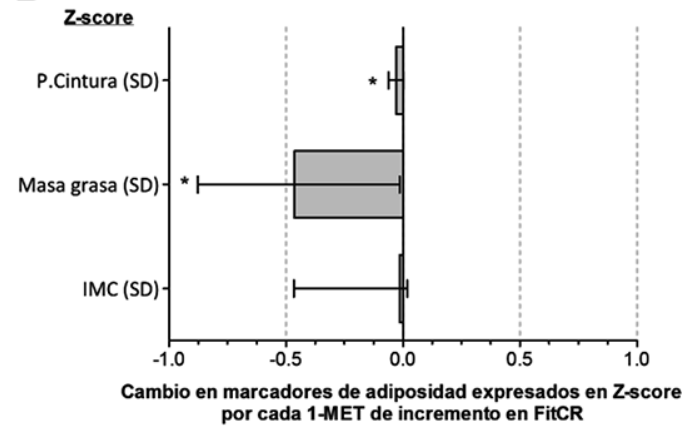

\section{C}

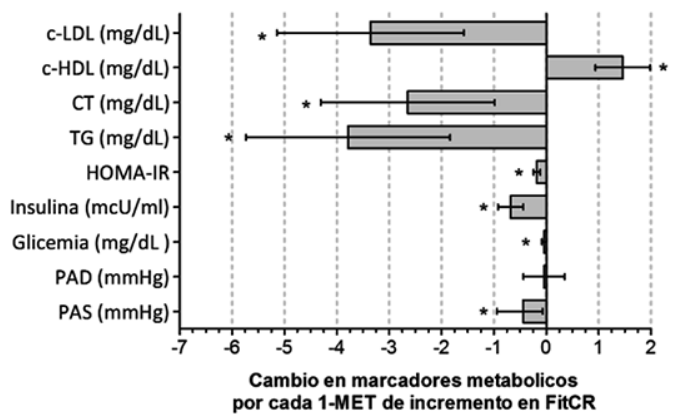

D

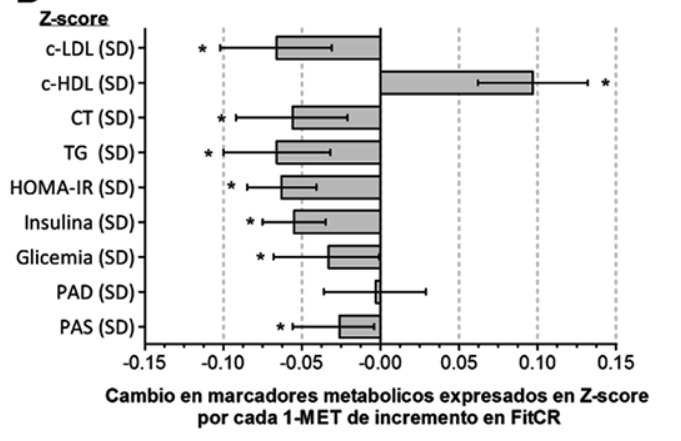

Figura 4. Estimación de la variación en marcadores antropométricos y metabólicos por cada 1-MET de incremento en FitCR. Valores expresados en unidades $\beta$ (gráficos A y C) y como Z-score (gráficos B y D). Todos los análisis fueron ajustados por edad, nivel educacional, sexo, hábito de fumar e IMC (para variables metabólicas). IMC: índice de masa corporal; PA: presión arterial; HOMA-IR: índice de resistencia insulínica; TG: triglicéridos; HDL: colesterol HDL; LDL: colesterol LDL; CT: Colesterol Total. SD: desviación estándar.

la relación entre el FitCR y marcadores metabólicos vinculados a la génesis de diversas enfermedades $^{23,24}$. La Monte et al., (2005) analizaron a más de 10.000 participantes y demostraron que un bajo nivel de FitCR es un fuerte predictor de incidencia de síndrome metabólico (SM) tanto en hombres como en mujeres ${ }^{23}$. Por otra parte, Zacardi et al., (2015) en una cohorte longitudinal de 23 años que incluyó a 2.520 hombres de mediana edad (53 años) demostró que un bajo nivel de FitCR está vinculado a un aumento de riesgo de diabetes mellitus tipo 2 (DM2), siendo estos resultados independientes a otros factores de riesgo ${ }^{24}$. Si bien nuestro estudio no incluye DM2o SM como las principales variables de estudio, si reportamos una asociación inversa entre FitCR y marcadores metabólicos vinculados a esta enfermedad y síndrome, respectivamente, como lo son glicemia, insulina, HOMA-IR y perímetro de cintura.
Cada uno de los estudios mencionados anteriormente ${ }^{23,24}$, nos entregan una clara señal de lo importante que es tener niveles adecuados de FitCR para conservar la salud de la población. Es por esta razón que últimamente, tanto a nivel clínico como a nivel de salud pública,se ha centrado la mirada en buscar estrategias que ayuden a mantener y/o aumentar el FitCR, independiente de cambios sobre el peso corporal, IMC, perímetro de cintura, etc. ${ }^{25}$, con el fin de reducir la génesis de diversas enfermedades cardiometabólicas y mortalidad de la población ${ }^{10,13,26,27}$.

La guía para la prescripción del ejercicio físico del Colegio Americano de Medicina Deportiva expone que es necesario cierta dosificación de ejercicio físico para lograr mantener o desarrollar el FitCR ${ }^{1}$. Si se considera que una parte importante de los chilenos no realiza actividad física de forma regular y que además, nuestro país presenta una 
tasa de sedentarismo que se ha incrementado de forma regular en los últimos 20 años ${ }^{3}$, podría suponerse que la disminución del FitCR estaría relacionada de forma importante con el aumento de las ECNT y ECVs en la población. Cabe mencionar que ambos componentes (sedentarismo e inactividad física) han demostrado influir negativamente sobre el FitCR ${ }^{5}$, por lo tanto, el estilo de vida hipocinético de los adultos chilenos provocaría que no se esté alcanzando el umbral fisiológico mínimo diario necesario para mantener el FitCR.

Tras lo señalado, es importante proponer una meta en el ámbito de la salud pública que sea realista y que ayude a direccionar los esfuerzos de la inclusión del ejercicio físico como herramienta preventiva y terapéutica para ciertas ECNT ${ }^{6}$. Un aumento de 1-MET en el FitCR es una propuesta prudente que la mayoría de las personas podrían $\operatorname{lograr}^{28}$. 1-MET ha sido documentado ampliamente en diversos estudios y ha demostrado un efecto importante tanto en la prevención de ECVs como en la reducción de la tasa de mortalidad ${ }^{10,29}$. En el presente estudio, de tipo transversal, seencontróuna relación inversa entre c-LDL, TG, HOMA-IR y perímetro de cintura por cada 1-MET de incremento en el FitCR.

Si bien es cierto que un aumento en los niveles de actividad física en la población chilena se ha relacionado con una disminución significativa de diversos factores de $\mathrm{ECVs}^{3}$, es importante señalar que el aumento del FitCR posee un efecto aún mayor ${ }^{28}$. Con el fin de optimizar los recursos económicos y el tiempo de las personas, sería ideal que los especialistas en salud no sólo las motiven a realizar ejercicio físico de forma regular, sino que además, promuevan el aumento de la intensidad del ejercicio con el fin de elevar el FitCR en un menor período de tiempo ${ }^{30,31}$. Este indicador de la magnitud del esfuerzo ha demostrado modular fuertemente la respuesta fisiológica y molecular del organismo ${ }^{32}$, potenciando al doble el aumento del FitCR cuando el ejercicio se realiza a una alta intensidad en comparación a un entrenamiento tradicional realizado a una intensidad moderada ${ }^{31}$.

Finalmente, los resultados de este estudio deben considerarse dentro del marco de sus fortalezas y limitaciones. El presente estudio posee un diseño de corte transversal limitando el análisis de causalidad de las variables incluidas. Estudios clínicos aleatorizados controlados son necesarios para reforzar la propuesta de 1-MET como estrategia de salud. Por otro lado, si bienes cierto que los análisis realizados fueron ajustados a diversos factores confundidores (edad, sexo, nivel educacional, hábito de fumar e IMC), también es importante señalar que otros factores podrían afectar estas asociaciones (alimentación). Respecto a la evaluación del FitCR, el test empleado no es un test directo y mucho menos considerado un gold standard, no obstante, se ha reportado una buena correlación entre el Chester Step Test y métodos directos de VO2 $\max ^{15}$, y además ofrece la ventaja de ser un método de menor costo y de fácil aplicabilidad en terreno, lo cual facilitaría su utilización en campo clínico y atención primaria.

\section{Conclusión}

Altos niveles de FitCR se asocian inversamente con diversos marcadores de adiposidad y metabólicos vinculados a enfermedades cardiometabólicas, por tanto, sería recomendable orientar los esfuerzos, tanto a nivel clínico como en salud pública, para promover tanto la práctica regular de ejercicio físico como reducir el hábito sedentario de los chilenos con el fin de aumentar dicho indicador. Estudios de intervención son necesarios para establecer si 1-MET de incremento en FitCR podría ser una meta objetiva y razonable para mejorar significativamente el estado de salud de la población.

Agradecimientos: Se agradece la colaboración de Francisco Pérez, Natalia Ulloa y Carlos Calvo durante la recolección de datos para el estudio GENADIO. También se agradece la contribución de Jason Gill y Mark Bailey a la concepción y diseño de este estudio de investigación.

\section{Referencias}

1. Garber CE, Blissmer B, Deschenes MR, Franklin BA, Lamonte MJ, Lee I-M, et al. American College of Sports Medicine position stand. Quantity and quality of exercise for developing and maintaining cardiorespiratory, musculoskeletal, and neuromotor fitness in apparently healthy adults: guidance for prescribing exercise. Med Sci Sports Exerc 2011; 43 (7): 1334-59.

2. Hallal PC, Andersen LB, Bull FC, Guthold R, Haskell W, Ekelund U, et al. Global physical activity levels: surveil- 
lance progress, pitfalls, and prospects. Lancet 2012; 380 (9838): 247-57.

3. Celis-Morales C, Salas C, Alduhishy A, Sanzana R, Martínez MA, Leiva A, et al. Socio-demographic patterns of physical activity and sedentary behaviour in Chile: results from the National Health Survey 2009-2010. J Public Health (Oxf) 2015; en prensa.

4. Barreto SM, Miranda JJ, Figueroa JP, Schmidt MI, Munoz S, Kuri-Morales PP, et al. Epidemiology in Latin America and the Caribbean: current situation and challenges. Int J Epidemiol 2012; 41 (2): 557-71.

5. Kulinski JP, Khera A, Ayers CR, Das SR, de Lemos JA, Blair SN, et al. Association between cardiorespiratory fitness and accelerometer-derived physical activity and sedentary time in the general population. Mayo Clin Proc 2014; 89 (8): 1063-71.

6. Pedersen BK, Saltin B. Exercise as medicine - evidence for prescribing exercise as therapy in 26 different chronic diseases. Scand J Med Sci Sports 2015; 25 (Suppl 3): $1-72$.

7. Fiuza-Luces C, Garatachea N, Berger NA, Lucia A. Exercise is the real polypill. Physiology (Bethesda) 2013; 28 (5): 330-58.

8. Russell AP, Foletta VC, Snow RJ, Wadley GD. Skeletal muscle mitochondria: a major player in exercise, health and disease. Biochim Biophys Acta 2014; 1840 (4): 127684.

9. Sharma S, Merghani A, Mont L. Exercise and the heart: the good, the bad, and the ugly. Eur Heart J 2015; 36 (23): 1445-53.

10. Kodama S, Saito K, Tanaka S, Maki M, Yachi Y, Asumi $\mathrm{M}$, et al. Cardiorespiratory fitness as a quantitative predictor of all-cause mortality and cardiovascular events in healthy men and women: a meta-analysis. JAMA 2009; 301 (19): 2024-35.

11. Blair SN. Physical inactivity: the biggest public health problem of the 21st century. Br J Sports Med 2009;43 (1): 1-2.

12. Hainer V, Toplak H, Stich V. Fat or fit: what is more important? Diabetes Care 2009; 32 (suppl 2): S392-7.

13. Berry JD, Willis B, Gupta S, Barlow CE, Lakoski SG, Khera A, et al. Lifetime risks for cardiovascular disease mortality by cardiorespiratory fitness levels measured at ages 45, 55, and 65 years in men. The Cooper Center Longitudinal Study. J Am Coll Cardiol 2011; 57 (15): 1604-10.

14. Celis-Morales CA, Pérez-Bravo F, Ibañes L, Sanzana $\mathrm{R}$, Hormazabal E, Ulloa $\mathrm{N}$, et al. Insulin resistance in Chileans of European and indigenous descent: evidence for an ethnicity $\mathrm{x}$ environment interaction. PLoS ONE 2011; 6 (9): e24690.
15. Buckley JP, Sim J, Eston RG, Hession R, Fox R. Reliability and validity of measures taken during the Chester step test to predict aerobic power and to prescribe aerobic exercise. Br J Sports Med 2004; 38 (2): 197-205.

16. Freedson PS, Melanson E, Sirard J. Calibration of the Computer Science and Applications, Inc. accelerometer. Med Sci Sports Exerc 1998; 30 (5): 777-81.

17. Marfell-Jones MJ, Stewart AD, de Ridder JH. International standards for anthropometric assessment. Potchefstroom, South Africa: The International Society for the Advancement of Kinanthropometry (ISAK); 2006.

18. Durnin JVGA, Womersley J. Body fat assessed from total body density and its estimation from skinfold thickness: measurements on 481 men and women aged from 16 to 72 years. British Journal of Nutrition 1974; 32 (1): 77-97.

19. Friedewald WT, Levy RI, Fredrickson DS. Estimation of the concentration of low-density lipoprotein cholesterol in plasma, without use of the preparative ultracentrifuge. Clin Chem 1972; 18 (6): 499-502.

20. Matthews DR, Hosker JP, Rudenski AS, Naylor BA, Treacher DF, Turner RC. Homeostasis model assessment: insulin resistance and beta-cell function from fasting plasma glucose and insulin concentrations in man. Diabetologia 1985; 28 (7): 412-9.

21. Celis-Morales CA, Perez-Bravo F, Ibañez L, Salas C, Bailey MES, Gill JMR. Objective vs. self-reported physical activity and sedentary time: effects of measurement method on relationships with risk biomarkers. PLoS ONE 2012; 7 (5): e36345.

22. Krämer V, Acevedo M, Orellana L, Chamorro G, Corbalán R, Bustamante MJ, et al. [Association between cardiorespiratory fitness and cardiovascular risk factors in healthy individuals]. Rev Med Chile 2009; 137 (6): 737-45.

23. LaMonte MJ, Barlow CE, Jurca R, Kampert JB, Church TS, Blair SN. Cardiorespiratory fitness is inversely associated with the incidence of metabolic syndrome: a prospective study of men and women. Circulation 2005; 112 (4): 505-12.

24. Zaccardi F, O'Donovan G, Webb DR, Yates T, Kurl S, Khunti $\mathrm{K}$, et al. Cardiorespiratory fitness and risk of type 2 diabetes mellitus: A 23-year cohort study and a meta-analysis of prospective studies. Atherosclerosis 2015;243 (1): 131-7.

25. King NA, Hopkins M, Caudwell P, Stubbs RJ, Blundell JE. Beneficial effects of exercise: shifting the focus from body weight to other markers of health. Br J Sports Med 2009; 43 (12): 924-7.

26. Lee D, Artero EG, Sui X, Blair SN. Mortality trends in the general population: the importance of cardiorespi- 
ratory fitness. J Psychopharmacol (Oxford) 2010; 24 (4 Suppl): 27-35.

27. Barry VW, Baruth M, Beets MW, Durstine JL, Liu J, Blair SN. Fitness vs. fatness on all-cause mortality: a meta-analysis. Prog Cardiovasc Dis 2014; 56 (4): 382-90.

28. Kaminsky LA, Arena R, Beckie TM, Brubaker PH, Church TS, Forman DE, et al. The importance of cardiorespiratory fitness in the United States: the need for a national registry: a policy statement from the American Heart Association. Circulation 2013; 127 (5): 652-62.

29. Nes BM, Vatten LJ, Nauman J, Janszky I, Wisløff U. A simple nonexercise model of cardiorespiratory fitness predicts long-term mortality. Med Sci Sports Exerc 2014; 46 (6): 1159-65.

30. Cristi-Montero C. [Is it enough to recommend to patients take a walk? Importance of the cadence]. Nutr Hosp 2013; 28 (4): 1018-21.

31. Weston KS, Wisløff U, Coombes JS. High-intensity interval training in patients with lifestyle-induced cardiometabolic disease: a systematic review and meta-analysis. Br J Sports Med 2014; 48 (16): 1227-34.

32. Laursen PB. Training for intense exercise performance: high-intensity or high-volume training? Scand J Med Sci Sports 2010; 20 (Suppl 2): 1-10. 